\title{
The in- or exclusion of non-breast cancer related death and contralateral breast cancer significantly affects estimated outcome probability in early breast cancer
}

\author{
R. A. Nout $\cdot$ W. E. Fiets $\cdot$ H. Struikmans $\cdot$ F. R. Rosendaal $\cdot$ \\ H. Putter $\cdot$ J. W. R. Nortier
}

Received: 27 June 2007 / Accepted: 9 July 2007 / Published online: 28 July 2007

(C) Springer Science+Business Media B.V. 2007

\begin{abstract}
A wide variation of definitions of recurrent disease and survival are used in the analyses of outcome of patients with early breast cancer. Explicit definitions with details both on endpoints and censoring are provided in less than half of published studies. We evaluated the effects of various definitions of survival and recurrent disease on estimated outcome in a prospectively determined cohort of 463 patients with primary breast cancer. Outcome estimates were determined both by the Kaplan-Meier and a competing risk method. In- or exclusion of contralateral breast cancer or non-disease related death in the definition of recurrent disease or survival significantly affects estimated outcome probability. The magnitude of this finding was dependent on patient-, tumour-, and treatment characteristics. Knowledge of the contribution of non-disease related death or contralateral breast cancer to estimated
\end{abstract}

R. A. Nout $(\bowtie) \cdot$ J. W. R. Nortier

Department of Clinical Oncology, Leiden University Medical Center, P.O. Box 9600, 2300 RC Leiden, The Netherlands

e-mail: r.a.nout@lumc.nl

W. E. Fiets

Department of Internal Medicin, Medical Center Leeuwarden, Leeuwarden, The Netherlands

H. Struikmans

Department of Radiation Oncology, Haaglanden Medical Center,

The Hague, The Netherlands

F. R. Rosendaal

Department of Clinical Epidemiology, Leiden University

Medical Center, Leiden, The Netherlands

H. Putter

Department of Medical Statistics and Bioinformatics, Leiden University Medical Center, Leiden, The Netherlands recurrent disease rate and overall death rate is indispensable for a correct interpretation and comparison of outcome analyses.

Keywords Breast cancer · End-points · Prognosis · Study design $\cdot$ Survival analysis

\section{Introduction}

In studies on early breast cancer, outcome is usually defined as the time from diagnosis or surgery until a particular event of interest (endpoint). The event of interest can vary and may include death (overall survival), disease related death (disease specific survival), or recurrent disease (disease free survival).

Altman et al. systematically reviewed the appropriateness of the application and presentation of survival analysis in clinical oncology journals [1]. They found that among papers specifically dealing with death as an endpoint; only $47 \%$ explicitly described this end-point as either any death or only cancer-related death. In as much as $61 \%$ of papers that studied time to progressive disease the handling of non-cancer related mortality was not clearly defined.

In studies on patients with early breast cancer a wide variation of definitions of disease free survival have been used. These definitions always included local recurrence, regional recurrence, and distant metastasis, but sometimes also included non-disease related death, contralateral breast cancer and in some cases second primary cancer. For example, the 1998 overview of randomised trials on adjuvant therapy included contralateral breast cancer in the analysis of disease recurrence, but did not include non-disease related death [2]. In the NSABP B14 
and B20 trials both non-disease related death, contralateral breast cancer, and second primary cancer were included as events in the definition of disease free survival $[3,4]$. In a recent report with long-term findings the definition of recurrence free survival was restricted to local or regional recurrence, or distant metastasis only [5].

Despite these different definitions, many papers on breast cancer survival do not provide an explicit definition of recurrent disease. A review on prognostic factors in node-negative breast cancer was published in 2002 by Mirza et al. [6]. In the methods section of their report, they stated that only papers in which overall or disease free survival were specified were included in their review. Sixty-three papers from their reference list dealt with survival analysis in primary breast cancer. We reviewed the definitions of recurrent disease used in these 63 papers. In only 21 out of 47 papers that studied time to recurrent disease the definition of recurrent disease explicitly described the handling of non-cancer related mortality. Intercurrent deaths were censored in 14 papers and counted as events in 7 papers. Eight papers explicitly described the handling of contralateral breast cancer. Contralateral breast cancer was censored in 1 and considered as event in 7 papers. The handling of second primary cancer was described in 7 papers. Second primary cancer was censored in 2 and counted as event in 5 papers.

In most papers the survival probability is estimated with the Kaplan-Meier method from observed survival times, censored or uncensored [7]. Censoring may arise due to end of follow-up, loss to follow-up, but also due to a competing event. The Kaplan-Meier method requires noninformative censoring, which means that those individuals who are censored should be as likely to have the subsequent event of interest as those who remain in the study. In particular competing events, such as: contralateral breast cancer or non-disease related death, might cause informative censoring. For this reason others have propagated an approach that accounts for informative censoring in survival analyses in the presence of competing events, a competing risk analysis [8-11].

The main objective of this study is to analyse the effect of various definitions of survival and relapse on prediction of outcome in patients with early breast cancer. Thereby, we focus on the influences of non-disease related death and contralateral breast cancer. Data from a (prospectively determined) cohort of 463 patients with primary breast cancer were used to evaluate the effects of various definitions of survival and relapse. A second objective was to compare the value of competing risk analysis with that to that of the Kaplan-Meier method for survival analysis.

\section{Material and methods}

Between October 1989 and March 1993463 patients diagnosed with operable, stage I to III breast cancer agreed to participate in a prospective registration study on prognostic factors. We obtained written informed consent from all patients. Surgery, radiotherapy and adjuvant systemic treatment were given according to the guidelines of the Comprehensive Cancer Centre Middle Netherlands. Patient-, tumour- and treatment characteristics are shown in Table 1. We assessed follow-up data until December 2002.

The events that were used to determine the different definitions of outcome were local- and regional recurrent disease, contralateral breast cancer, distant metastasis, disease related death and non-disease related death. In the various analyses these events were either ignored, considered as event of interest or as competing event (censored), depending on the various definitions of outcome. Definitions of overall survival, diseases specific survival, disease free interval, and disease free survival are given in Table 2. We defined local recurrent disease as either recurrence in the skin or soft tissue of the (ipsilateral) chest wall or in the ipsilateral breast. Regional recurrent disease confined recurrence in the lymph nodes in the ipsilateral axilla, infraclavicular fossa or internal mammary chain. Contralateral breast cancer included invasive breast cancer lesions in the contralateral breast regardless of histological type, lymph node involvement, and time interval from initial

Table 1 Patient-, tumour-, and treatment characteristics

Number of patients $(\%)$

\begin{tabular}{lc}
\hline Age & $142(31)$ \\
$\leq 50$ year & $213(46)$ \\
$51-70$ year & $108(23)$ \\
$>70$ year & $266(57)$ \\
Primary surgical therapy & $190(41)$ \\
Breast conserving therapy & $7(2)$ \\
Modified radical mastectomy & \\
Other & $142(31)$ \\
Adjuvant systemic therapy & $72(16)$ \\
Hormonal therapy & \\
Chemotherapy & $290(63)$ \\
Histology & $173(37)$ \\
Ductal & \\
Other & $272(59)$ \\
Tumour size & $191(41)$ \\
$\leq 20$ mm & \\
$>$ 20 mm & $278(60)$ \\
Axillary lymph nodes & $185(40)$ \\
Negative &
\end{tabular}


Table 2 Definitions of outcome.

\begin{tabular}{lc}
\hline Overall survival & Time from surgery until death from any cause \\
Disease specific survival & Time from surgery until death related to breast cancer. Death not related to breast cancer \\
& is censored (Kaplan-Meier analysis) or treated as competing event (competing risk analysis). \\
Disease free interval & Time from surgery until recurrent disease. ${ }^{\text {a }}$ Death not related to breast cancer is censored \\
(Kaplan-Meier analysis) or treated as competing event (competing risk analysis).
\end{tabular}

${ }^{a}$ In the definition of recurrent disease local recurrence, regional recurrence, and distant metastasis are considered events; contralateral breast cancer is ignored, treated as event or censored (Kaplan-Meier analysis) / treated as competing event (competing risk analysis)

therapy or from subsequent recurrent disease. Breast cancer lesions at any other site, including the ipsilateral supraclavicular lymph nodes, were classified as distant metastases. We classified death as disease related when death occurred in patients known with distant metastases. Otherwise we classified death as non-disease related.

Survival probabilities were determined both by KaplanMeier method [7], and by competing risk analyses [8]. Competing risk analyses were calculated as described in "Tutorial in biostatistics: competing risks and multi-state models" by Putter et al. [8].

\section{Results}

During median 10 years of follow-up 149 patients died. About 91 deaths were related to breast cancer, and another 58 patients died from causes unrelated to breast cancer. Local recurrences were diagnosed in 28 patients, regional recurrences in another 24. Distant metastases occurred in 111 patients, and in 30 patients breast cancer was diagnosed in the contralateral breast. Estimated with the Kaplan-Meier method, after 10 years of follow-up $68 \%$ of patients were still alive (overall survival). If no one had died from causes other than breast cancer, $79 \%$ of patients would have been alive (disease-specific survival) (Table 3). Disease free survival varied between $56 \%$ and $59 \%$, depending on the definition of relapse. If no patients had died during follow-up, 65-69\% of patients would have been free of recurrent disease (disease free interval) (Table 3). Compared with the competing risk approach, the Kaplan-Meier method slightly underestimated 10-year survival rates when one or more competing events were censored instead or ignored. The largest difference (2.0 percent-point) was found when both non-disease related death and contralateral breast cancer were censored (Table 3).

\section{Non-disease related death}

The difference in estimated survival probability between overall survival and disease specific survival and between disease free survival and disease free interval is by definition caused by the handling of non-disease related death. As older age is associated with a higher probability of nondisease related death, we evaluated the effect of patient's

Table 3 Estimated 10-year survival according to definition of survival determined both by Kaplan-Meier method and the competing risk analysis.

\begin{tabular}{|c|c|c|c|c|c|c|}
\hline \multirow[t]{3}{*}{ Survival definition } & \multicolumn{6}{|c|}{ 10-year Survival (\%) } \\
\hline & \multicolumn{2}{|c|}{ All patients } & \multicolumn{2}{|c|}{ No adjuvant systemic therapy } & \multicolumn{2}{|c|}{ Adjuvant systemic therapy } \\
\hline & KM & $\mathrm{CR}$ & KM & $\mathrm{CR}$ & $\mathrm{KM}$ & $\mathrm{CR}$ \\
\hline Overall survival & 68.0 & & 75.8 & & 58.6 & \\
\hline Disease specific survival & 79.3 & 80.6 & 85.3 & 86.2 & 71.9 & 73.7 \\
\hline \multicolumn{7}{|l|}{ Disease free survival } \\
\hline Contralateral $\mathrm{BC}$ ignored & 59.3 & & 65.8 & & 51.2 & \\
\hline Contralateral BC censored & 58.6 & 59.4 & 64.9 & 66.0 & 51.1 & 51.6 \\
\hline Contralateral BC event & 55.5 & & 59.9 & & 50.2 & \\
\hline \multicolumn{7}{|l|}{ Disease free interval } \\
\hline Contralateral $\mathrm{BC}$ ignored & 69.4 & 70.9 & 74.6 & 75.8 & 63.0 & 64.9 \\
\hline Contralateral BC censored & 68.9 & 70.9 & 73.9 & 75.9 & 63.2 & 65.4 \\
\hline Contralateral $\mathrm{BC}$ event & 64.8 & 66.5 & 67.6 & 69.2 & 61.3 & 63.4 \\
\hline
\end{tabular}

KM: Kaplan-Meier method; CR: competing risk analysis; BC: breast cancer 
age on estimated survival probability using the various definitions of survival. As shown in Table 4, patients aged more than 70 years were at risk for dying from a cause unrelated to breast cancer, whereas patients aged 50 years or less seldom died from a cause unrelated to breast cancer. As a consequence, in the younger subgroup 10-year overall survival was almost equal to 10 -year disease specific survival. Whereas in the elderly, estimated 10 -year disease specific survival was more than $30 \%$ point better than estimated 10-year overall survival (Fig. 1). In the younger subgroups differences between Kaplan-Meier and competing risk estimates were limited $(\leq 1 \%)$. In the elderly estimations of 10-year disease specific survival were $82.2 \%$ and $84.9 \%$ with Kaplan-Meier and competing risk analyses, respectively. Estimations of 10-year disease free interval were $73.6 \%$ and $77.6 \%$ respectively for two statistical methods.

\section{Contralateral breast cancer}

We evaluated the effect of the inclusion of contralateral breast cancer as event in the analysis of disease recurrence on estimated disease free interval and disease free survival (Table 3). The administration of adjuvant systemic therapy is known to reduce the risk of contralateral breast cancer $[12,13]$. In the whole study population the absolute reduction in disease free survival or disease free interval due to inclusion of contralateral breast cancer as event in the definition of relapse was approximately $4 \%$; in patients not treated with adjuvant systemic therapy 6-7\%, and in patients treated with adjuvant systemic therapy $1-2 \%$. In the broadest definition of relapse 197 events were counted during 10-years follow-up, including 47 non-disease related deaths and 26 contralateral breast cancers. That is, in the analysis of disease free interval $17 \%$ of events were contralateral breast cancers, compared with $13 \%$ in the analysis of disease free survival. Consequently, the effect of the inclusion of contralateral breast cancer as event in the definition of relapse was greater when estimating disease free interval than when estimating disease free survival (Table 3).

Similarly, the greatest effect of the inclusion of contralateral breast cancer and non-disease related death as events on estimated disease recurrence rate was found in patients with low risk breast cancer. In a subgroup of 168 patients with T1N0 breast cancer, not treated with adjuvant systemic therapy, the 10-year relapse rate including local relapse, regional relapse, or distant metastasis was $23 \%$. The estimated 10 -year relapse rate rose to $31 \%$ both with the inclusion of either contralateral breast cancer or nondisease related death as event in the definition of relapse, and to $38 \%$ with the inclusion of both events in the definition of relapse.

\section{Discussion}

In the present study we show that the inclusion of contralateral breast cancer or non-disease related death as event in the definition of recurrent disease or survival of early breast cancer significantly affects estimated outcome probability. The magnitude of the effect depends on patient-, tumour-, and treatment characteristics. The greatest effect was observed for the handling of non-disease related death within the group of patients older than 70 years, and for the inclusion of contralateral breast cancer as event in patients with low risk breast cancer not treated with adjuvant systemic therapy.

These findings stress the importance of a clear definition and reporting of outcome parameters in scientific papers presenting the results of phase III randomized trials. However, the magnitude of the observed effects can differ depending on the various events that have been included, ignored or censored in the definition of outcome. As a consequence, the in- or exclusion of contralateral breast cancer or non-disease related death in the definition of

Table 4 Estimated 10-year event rate according to age at diagnosis determined both by Kaplan-Meier method and competing risk analysis.

\begin{tabular}{|c|c|c|c|c|c|c|}
\hline \multirow[t]{3}{*}{ Event } & \multicolumn{6}{|c|}{10 -year Event rate $(\%)$} \\
\hline & \multicolumn{2}{|c|}{$\leq 50 \mathrm{yr}$} & \multicolumn{2}{|c|}{$51-70 \mathrm{yr}$} & \multicolumn{2}{|c|}{$>70 \mathrm{yr}$} \\
\hline & KM & $\mathrm{CR}$ & $\mathrm{KM}$ & $\mathrm{CR}$ & $\mathrm{KM}$ & $\mathrm{CR}$ \\
\hline Overall death & 31.1 & & 23.5 & & 52.0 & \\
\hline Disease related death & 28.6 & 28.1 & 16.4 & 15.7 & 17.7 & 15.1 \\
\hline Non-disease related death & 3.6 & 3.0 & 8.5 & 7.8 & 41.7 & 36.9 \\
\hline Recurrent disease or death & 41.5 & & 32.2 & & 58.7 & \\
\hline Recurrent disease & 39.5 & 38.8 & 26.8 & 25.8 & 26.3 & 22.4 \\
\hline Death without recurrent disease & 3.2 & 2.7 & 7.5 & 6.5 & 43.8 & 36.2 \\
\hline
\end{tabular}

KM: Kaplan-Meier method; CR: competing risk analysis. Recurrent disease was defined as either local recurrence, regional recurrence or distant metastasis whichever came first. Occurring contralateral breast cancer was ignored 


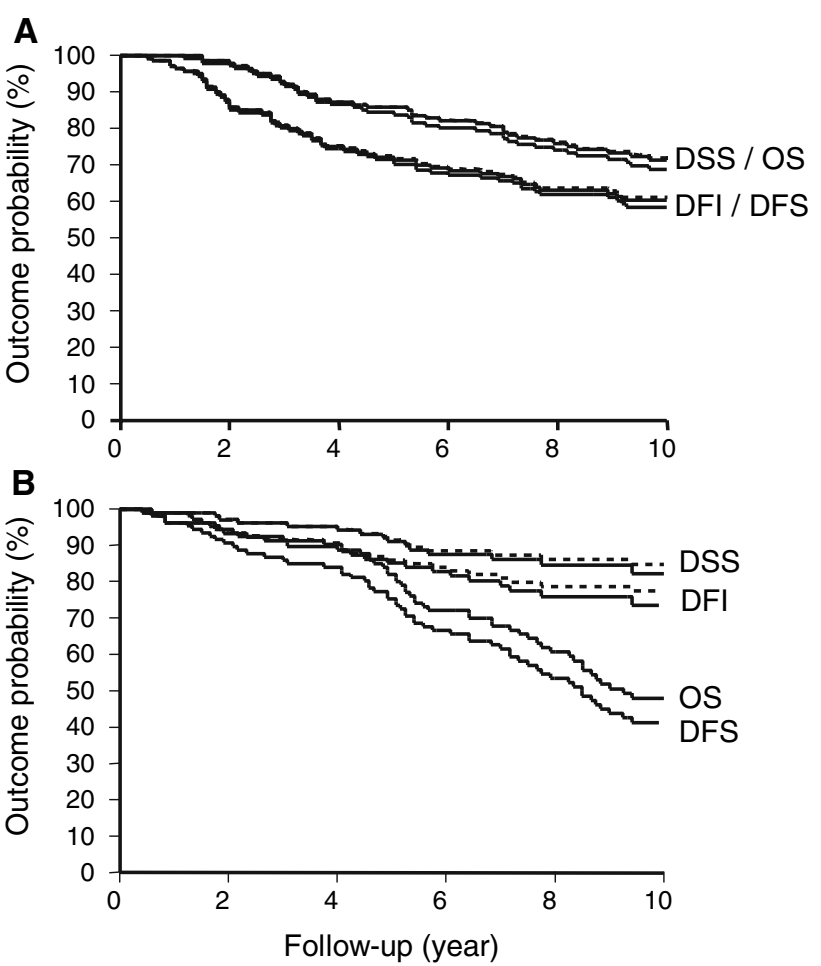

Fig. 1 Influence of survival definitions on estimated outcome probability in breast cancer patients 50 years or less of age (A), and over 70 years of age $(\mathbf{B})$. Both by Kaplan-Meier method (solid line) and competing risk analysis (dotted line). DSS: disease specific survival; OS: overall survival; DFI: disease free interval; DFS: disease free survival. Contralateral breast cancer was ignored in the definition of relapse.

outcome could influence the results of a trial. We can illustrate this with data from the Anastrozole, Tamoxifen, Alone or in Combination (ATAC) trial, in which 6241 patients were included in the 2 relevant arms of this trial $[12,13]$. After a median follow-up of 68 months, 831 patients have died (411 patients treated with anastrozole and 420 patients treated with tamoxifen). More patients who were treated with tamoxifen died from breast cancer than patients who were treated with anastrozole (265 vs. 235), whereas fewer patients who received tamoxifen died from a cause not related to breast cancer (155 vs. 176). Treatment with anastrozole also led to a reduction in disease recurrences (402 vs. 498). A considerable part of this reduction was caused by the difference in occurrence of contralateral breast cancers (35 vs. 59). Consequently, anastrozole led to an improvement in disease free survival (Hazard Rate (HR) 0.87, $P=0.01$ ), and an even better improvement in disease free interval (HR 0.79, $P=0.0005)$. Overall survival was similar for anastrozole and tamoxifen treated patients (HR 0.97), whereas disease specific survival was $12 \%$ better in the anastrozole group, although this was not significant (HR $0.88, P=0.20$ ).
These data from the ATAC trial illustrate that a clear definition of survival endpoints, including the contribution of non-disease related death and the contribution of contralateral breast cancer to the estimated disease recurrence rate are crucial for a correct interpretation of outcome analyses in clinical trials. These data also demonstrate that a significant difference in disease free survival is not automatically followed by a significant difference in overall survival.

The Kaplan-Meier method for estimating survival has repeatedly been criticised for possible biases in the estimation of event rates $[8,9,11,14]$. In the presence of competing events, cumulative incidence functions of the events of interest are probably evaluated more appropriately by taking into account other events within a competing risk framework. In general, event rates derived using the Kaplan-Meier approach are larger than estimates accounting for competing risks, $[8,9,11]$ and differences between Kaplan-Meier and competing risk approaches can become substantial when the competing risk event is related to or is a result of the underlying disease. But, as presented by Satagopan et al. ignoring the informative censoring mechanism does not substantially influence the estimates of breast cancer-specific mortality [15]. We present similar results in our estimations of disease-specific survival and disease free survival. However, differences became more substantial when relatively more patients were censored due to competing events. Although not the subject of our current analyses, it is probably more likely that ignoring of the informative censoring mechanism will substantially influence the estimates of local recurrence.

\section{Conclusion}

Clear definitions of endpoints and competing events are crucial for the correct interpretation and reliable comparison of outcome studies. In the present study on patients with early breast cancer, the inclusion of contralateral breast cancer and/or non-disease related death substantially influenced estimates of recurrent disease rate and survival, specifically in elder patients and patients with a good prognosis. Bias generated by the Kaplan-Meier approach due to informative censoring of contralateral breast cancer or non-disease related death was limited.

\section{References}

1. Altman DG, De Stavola BL, Love SB, Stepniewska KA (1995) Review of survival analyses published in cancer journals. Br J Cancer 72:511-518

2. Altman DG, De Stavola BL, Love SB, Stepniewska KA (1998) Polychemotherapy for early breast cancer: an overview of the 
randomised trials. Early Breast Cancer Trialists' Collaborative Group. Lancet 352:930-942

3. Fisher B, Costantino J, Redmond C, Poisson R, Bowman D, Couture J, Dimitrov NV, Wolmark N, Wickerham DL, Fisher ER, Wolmark N (1989) A randomized clinical trial evaluating tamoxifen in the treatment of patients with node-negative breast cancer who have estrogen-receptor-positive tumors. N Engl J Med 320:479-484

4. Fisher B, Dignam J, Wolmark N, DeCillis A, Emir B, Wickerham DL, Bryant J, Dimitrov NV, Abramson N, Atkins JN, Shibata H, Deschenes L, Margolese RG (1997) Tamoxifen and chemotherapy for lymph node-negative, estrogen receptor-positive breast cancer. J Natl Cancer Inst 89:1673-1682

5. Fisher B, Jeong JH, Bryant J, Anderson S, Dignam J, Fisher ER, Wolmark N (2004) Treatment of lymph-node-negative, oestrogen-receptor-positive breast cancer: long-term findings from national surgical adjuvant breast and bowel project randomised clinical trials. Lancet 364:858-868

6. Mirza AN, Mirza NQ, Vlastos G, Singletary SE (2002) Prognostic factors in node-negative breast cancer: a review of studies with sample size more than 200 and follow-up more than 5 years. Ann Surg 235:10-26

7. Kaplan EL, Meier P (1958) Nonparametric estimation from incomplete observations. J Am Stat Assoc 53:457-481

8. Putter H, Fiocco M, Geskus RB (2007) Tutorial in biostatistics: competing risks and multi-state models. Stat Med 26:2389-2430
9. Arriagada R, Rutqvist LE, Kramar A, Johansson H (1992) Competing risks determining event-free survival in early breast cancer. Br J Cancer 66:951-957

10. Broet P, de la RA, Scholl SM, Fourquet A, Mosseri V, Durand JC, Pouillart P, Asselain B (1995) Contralateral breast cancer: annual incidence and risk parameters. J Clin Oncol 13:1578-1583

11. Chen Y, Thompson W, Semenciw R, Mao Y (1999) Epidemiology of contralateral breast cancer. Cancer Epidemiol Biomarkers Prev 8:855-861

12. Baum M, Budzar AU, Cuzick J, Forbes J, Houghton JH, Klijn JG, Sahmoud T (2002) Anastrozole alone or in combination with tamoxifen versus tamoxifen alone for adjuvant treatment of postmenopausal women with early breast cancer: first results of the ATAC randomised trial. Lancet 359:2131-2139

13. Howell A, Cuzick J, Baum M, Buzdar A, Dowsett M, Forbes JF, Hoctin-Boes G, Houghton J, Locker GY, Tobias JS (2005) Results of the ATAC (Arimidex, Tamoxifen, alone or in combination) trial after completion of 5 years' adjuvant treatment for breast cancer. Lancet 365:60-62

14. Gelman R, Gelber R, Henderson IC, Coleman CN, Harris JR (1990) Improved methodology for analyzing local and distant recurrence. J Clin Oncol 8:548-555

15. Satagopan JM, Ben Porat L, Berwick M, Robson M, Kutler D, Auerbach AD (2004) A note on competing risks in survival data analysis. Br J Cancer 91:1229-1235 